\title{
Families and Genetic Diagnoses in the Genomic and Internet Age
}

\author{
Debra Skinner, PhD; Rebecca Schaffer, BA
}

\begin{abstract}
The explosion of new knowledge and technologies stimulated by the Human Genome Project has ushered in the "genomic age," where genetic components of diseases and disorders, their links, and processes are increasingly being revealed and understood at the molecular level. The genomic age coupled with the Internet, which offers quick access and circulation of information as well as numerous sites in which "knowledge" can be recreated or disputed, is changing the contexts and experiences of early childhood disability. This article examines this transformation using data from an ethnographic, longitudinal study of parents of young children with suspected or known genetic disorders. Here we describe how genetic information and the Internet affect parents' search for and understandings of genetic diagnoses, and how these 2 cultural forces may be changing medical, early intervention, and special education practices. Key words: culture, genetic disorder, Internet, medical practice, parents
\end{abstract}

$\mathbf{K}$ NOWLEDGE and practices of early intervention form and evolve in specific sociohistorical contexts. What is known about early childhood disability and responses to it are shaped by multiple influences, including biomedical and educational research and technology, as well as policies and advocacy efforts. As these larger contexts change, systems and services of early intervention must also adapt. Two forces that have major implications for early intervention services have emerged recently. One is the explosion of new knowledge and technologies stimulated by the Human Genome Project. The other is the vast realm of information and communication available through the Internet.Their

From FPG Child Development Institute (Dr Skinner) and the Department of Anthropology (Ms Schaffer), University of North Carolina at Chapel Hill, Chapel Hill.

The Culture and Family Interpretations of Genetic Disorder Project was funded by a grant (1 RO1 HG02164) from the Ethical, Legal, and Social Implications (ELSI) Research Program/NIH/NHGRI.

Corresponding autbor: Debra Skinner, PhD, Frank Porter Grabam Child Development Institute, CB \#8180, University of North Carolina at Chapel Hill, Chapel Hill, NC 27599 (e-mail: skinner@mail.fpg.unc.edu) convergence has resulted in countless Web sites devoted to expert and lay knowledge of specific genetic disorders and to advocacy and support groups formed around them. Now information related to genetic disorders, their associated conditions, courses of treatment, and support communities can be quickly accessed and circulated online. This intersection of knowledge and media constitutes a powerful social context that has begun to change families' experiences of early childhood disability and their interactions with those who provide medical, therapeutic, and educational interventions.

Speculation has begun about what recent advances in genetic knowledge and technologies mean for early intervention and special education services while some limited research on the impact of the Internet on families' decisions regarding services has also been carried out, the consequences of the confluence of genomics and the Internet for families and service providers are yet to be addressed. After reviewing the relevant literature, we report findings from a longitudinal, ethnographic study on the ways in which online genetic information has affected the understandings and actions of parents (or 
potential parents) of children with known or suspected genetic disorders.

\section{THE GENOMIC ERA}

The Human Genome Project, begun in 1990 as an international effort to produce a comprehensive sequence of the human genome, has led to discoveries of the genetic basis for hundreds of disorders and advanced understandings of basic genetic processes at the molecular level (Emilien, Ponchon, Caldas, Isacson, \& Maloteaux, 2000; Falcon de Vargas, 2002). Technologies that locate the genetic basis of disorders have also increased dramatically, with hundreds of genetic tests available in research or clinical practice and many more to come (Yoon et al., 2001). A primary goal of these efforts is to translate genome-based science into new and more effective strategies for the prevention, diagnosis, and treatment of diseases and disorders (Collins, Green, Guttmacher, \& Guyer, 2003).

These advances have captured the public and scientific imagination, resulting in a barrage of works on genetics in the last decade, many of which characterize this as the "age of genomics" (Condit, 1999; Conrad \& Gabe, 1999; Nelkin \& Lindee, 1995). Provocative metaphors used to describe the mapping of the genome include "a search for the 'holy grail,' investigating 'the essence of human life,' and decoding 'the book of life"' (Conrad \& Gabe, 1999, p. 2). These popular metaphors present optimistic views of the explanatory powers and the beneficial potential of genetic research. While social science analyses are more cautionary in tone, there is little doubt that new genetic knowledge and technology, and their commercialization, will change both professional and parental understandings of genetic disorders and the ways in which services are delivered (Khoury, McCabe, \& McCabe, 2003).

Bailey and Powell (in press) have noted potential ways that both families and practitioners may be impacted by the explosion of genetic knowledge. As parents and practitioners begin to learn more about genetic disorders, they will need (1) more comprehensible information about genetics in general and specific disorders in particular; (2) the ability to speak the basic language of genetics; (3) access to a wide range of information and support, including genetic counseling and parent support groups related to a particular disorder; (4) assistance in sorting through information of varying quality and authority; and (5) assistance in determining the most effective treatment for a particular disorder, including pharmacological approaches, alternative or controversial treatments, or possible cures.

Hodapp and colleagues (Hodapp, DesJardin, \& Ricci, 2003; Hodapp \& Fidler, 1999) have focused specifically on this question of etiology-based interventions, noting the link of specific genetic disorders (eg, Down syndrome, fragile $\mathrm{X}$ syndrome, PraderWilli syndrome, and Williams syndrome) with a heightened probability or likelihood of a particular behavioral phenotype. Although they recognize variability within a disorder, they predict that etiology-based strengths and weaknesses will suggest specific and targeted intervention practices and that early interventionists and special educators will need to have information about various treatment options thought to be most effective for the behavioral and cognitive profile commonly associated with a specific disorder, such as fragile $\mathrm{X}$ syndrome. Others have disagreed with this view of etiology-based services, noting variations within and commonalities across syndromes and the numerous problems associated with a proliferation of categories (Forness \& Kavaale, 1994). While professionals debate best practices, the data we present below indicate that parents of children with genetic diagnoses are turning to the Internet for information on diagnosis-specific treatments and interventions and developing their own perspectives on this issue.

\section{THE INTERNET AGE AND CYBERGENETICS}

The supply of and demand for online health resources (cybermedicine or e-health) 
have skyrocketed over the last decade (Baker, Wagner, Singer, \& Bundorf, 2003; Cotten \& Gupta, 2004; CyberAtlas, 2001; Fox \& Fallows, 2003; Gerber \& Eiser, 2001; Health on the Net Foundation, 1999). The small but growing literature on cybergenetics indicates that families are taking advantage of these online resources, searching for genetic information to prepare for visits to genetic clinics or to interpret information from their visits to these clinics (Christian, Kieffer, \& Leonard, 2001; Marion \& Mendelson, 2000; Taylor, Alman, \& Manchester, 2001). Moreover, as parents publish their children's case histories and share advice in chat rooms, on listservs, and via personal home pages, they take part in increasingly influential productions of genetic knowledge (Solovitch, 2001).

Studies of the consequences of e-health use have only just begun, but a growing body of theory, supported by some empirical evidence, suggests that the Internet can enhance patient-doctor relationships by providing a common vocabulary, aligning expectations, and increasing patients' confidence in doctors' conclusions and advice (Baker et al., 2003; Fox \& Fallows, 2003; Gerber \& Eiser, 2001; Hardey, 1999, 2000; Potts \& Wyatt, 2002; Solovitch, 2001). These studies also indicate that the Internet may empower users by providing them with the scientific literacy, personal validation, and social support networks they need to become proactive partners with the medical establishment. Moreover, Hardey (2000) argues that e-health users who share their experiences with others online take part in a process that Giddens (1991) has described as the "reappropriation" of health expertise, a process through which e-health users come to see their own experiential knowledge as both valid and important. Hardey argues that the interweaving of personal experiences, lay and scientific knowledge, and advice on personal home pages, listservs, and other online resources represents "a shared production and exploration of solutions, support, remedies, and lifestyles that has not previously been possible" (p. 2). As e-health users' scientific and lay knowledge grow, they may come to see medical profes- sionals as one of many sources of authoritative knowledge and themselves as critical information managers who must seek out and weigh all of the information and options available to them (Fox \& Fallows, 2003; Hardey, 1999; Solovitch, 2001; Ziebland, 2004).

While it is clear that the Internet has provided easier and broader access to a wide range of genetic and medical information, to date there has been little empirical research on the effect this may be having on parents' uses of genetic information and, in turn, on their interactions with health and other service providers. Here we present findings from the Culture and Family Interpretations of Genetic Disorder Project, a recently completed ethnographic study of 106 families referred to a pediatric genetic counseling and evaluation clinic that provides evidence of what the confluence of genetic discoveries and the Internet means for families and providers.

\section{THE STUDY}

The overall aim of the project was to examine how parents from different ethnic and cultural backgrounds who had a child or who were at risk for having a child with a genetic disorder sought out, understood, and used information (including information received during genetic counseling and evaluation sessions) to interpret genetic disorders and to make decisions about reproduction, health, and services. We recruited families who were referred by their healthcare providers or developmental evaluation centers to a pediatric genetic clinic in the southeastern United States. This clinic, which is associated with a university-based hospital, provides medical genetic counseling and evaluation services for the state's citizens and their physicians through evaluation and diagnosis of genetic and metabolic disorders in children and adults, counseling these clients and their families, and providing long-term medical management and follow-up care when appropriate (as in the case of disorders such as phenylketonuria and Prader-Willi syndrome).

We purposively recruited families who represented the ethnic and socioeconomic 
diversity of the clinic population. A few families were biracial or multiracial, but of the 106 mothers or other primary informants in the families, 23 identified themselves as African American, 62 European American, 13 Latino, and 8 Native American. All but one of the Latino families were from either Mexico or Puerto Rico. Half of these families had been living in the United States for 10 years or more, and the others had moved to the United States within the last 3 years, 2 specifically to find services for their children. Families represented a full range of income and education levels. Forty-seven percent had annual incomes of less than $\$ 30,000$, while $21 \%$ had annual incomes of more than $\$ 75,000$. Only $7 \%$ of the mothers had less than a high school education, $26 \%$ had a high school diploma or GED, 34\% had some college or an associate's degree, $23 \%$ had a bachelor's degree, and $10 \%$ had advanced degrees. Children referred to the clinic ranged in age from 1 month to 18 years, but $65 \%$ were 5 years of age or younger. The average age of this group of young children was 30 months. An important variable for the purposes of our study was whether or not families received a definitive diagnosis of a genetic condition. In this sample of 106, 44 families did not get a clear diagnosis and 62 families received or had confirmed a definitive genetic diagnosis through genetic testing or a clinical examination.

The longitudinal, ethnographic study began with families' first visit to the clinic. Ethnographers observed and wrote extensive field notes on family members' and clinicians' communications and interactions during the evaluation and counseling session (or sessions if a family returned), and followed this observation with 4 in-depth, semistructured interviews with each family completed every 6 months. Interview questions covered a range of topics, including parents' perspectives on and evaluation of the clinic visit and any testing procedures; where they searched for information, services, and supports in relation to the child's condition; their understandings of the condition and its ramifications; family routines and accommodations; deci- sions made related to health, reproduction, and services; and any changes in these areas over time. Interviews lasted for approximately 90 minutes and were typically conducted in the home or other place of the family's choice. (We conducted telephonic interviews with families that moved during the study.) Audio recordings of the interviews were transcribed verbatim and translated from Spanish to English when necessary. Through an iterative process (Miles \& Huberman, 1994; Skinner, Rodriguez, \& Bailey, 1999), the research team developed a coding scheme that was used to link analytic or content categories (ie, codes) to text segments in the transcriptions and field notes. The coded text was then entered into a software program (QSR4) that can index (ie, attach codes), search, and retrieve sections of data related to particular topics. For this analysis, we searched for all data coded for parents' use of the Internet. We then created matrices (Miles \& Huberman, 1994), which allowed us to systematically categorize and compare how parents used the Internet and the outcomes of this use. Of the 106 families, 100 provided information about their use of online health resources. These are the families we report on below.

\section{FINDINGS}

\section{Prevalence}

Of the 100 families in this study, 83 had used the Internet for some purpose related to their child's condition. Of these 83 families, 69 went online themselves and another 14 had others search the Internet for them. Almost all the European American families (94\%) used e-health resources, followed by Native American (86\%), African American (65\%), and Latino (55\%) families. Although use was lower in these latter groups, this was largely due to differences in family income and education levels. Overall, families of any ethnicity with lower income and lower education levels were less likely to use the Internet, but voracious users existed even among these families. Of the families with annual household incomes below \$30,000, 69\% reported using 
the Internet. Two thirds of the parents with high school education or less were e-health users. Prevalence of usage also varied within the family. In the vast majority of families, the mother was the primary or sole user of online health resources.

\section{Reasons for Internet use}

Families used the Internet for multiple purposes, which varied over time. Several mothers turned to the Internet prior to obtaining a diagnosis for their child's condition, plugging in the child's symptoms and characteristics in an effort to diagnose the disorder themselves or to identify appropriate medical specialists who could do so. For example, one mother of 2 children with multiple physical and behavioral problems researched disorders that matched their symptoms and then contacted specialists who she thought could provide a diagnosis. When a pediatric geneticist diagnosed her children with hypochondroplasia, she returned to the Internet to search for behavioral therapies appropriate for this condition. She stated, "That's how most of this has come about is through me doing my own research. I've been my own advocate for the girls. I've done a lot of Internet studies."

Another mother reported that she had a difficult time getting doctors to take seriously her concerns about her daughter's developmental delays, small size, and mini-seizures until she began going online to identify potential diagnoses. By her account, this enabled her to acquire a basic understanding of medical terminology and procedures that she used to communicate more effectively with her doctors, and gain the referral for diagnostic testing that she desired. While this mother did not obtain a definitive genetic diagnosis, she managed to rule out several potential diagnoses and found information that led her to symptom-specific services and treatments for her child. She also achieved good working relationships with doctors and other specialists, both online and offline, who she believed would assist in her ongoing efforts to obtain a diagnosis. Other mothers noted that when they demonstrate scientific literacy at the be- ginning of a clinic visit, medical professionals treat them as more knowledgeable clients, going into more detail about genetic conditions and their possible ramifications. The Internet served this critical educational role not only during the quest for a diagnosis but also in subsequent efforts to identify and access the most appropriate services and treatments for the child's condition.

The majority of e-health users in this study did not turn to the Internet until they received a potential or confirmed diagnosis. This gave them a term to plug into a search engine. A few parents said they were too overwhelmed or did not feel comfortable asking questions during the clinic session, and so they relied on the Internet to answer their questions or clear up any confusion. They searched for resources that would provide explanations in layman's terms, prognoses, testimonials or case descriptions by other families having children with the same diagnosis, and treatment options. Once they were confident of their own understanding of the condition, they often shared online resources with their relatives and their children's healthcare providers and educators.

While most parents with a confirmed diagnosis tended to use the Internet intensively at first and then less frequently after they developed a solid understanding of the condition and treatment options, about 10\% remained avid users even after conducting extensive online research. These parents often became active participants in online support and advocacy groups, keeping close tabs on the latest research, comparing their child's medical history and progress to that of other children with the same or similar conditions, sharing advice on a wide range of topics, and advocating for specific types of research and treatment. These avid users were either families of children with extremely rare genetic conditions or families who searched the Internet to explore "second opinions" on treatment plans.

Parents of children with rare disorders turned to the Internet to locate other families having children with the same diagnosis. 
Several mothers said that they wanted to know that they were not "the only ones," that they wanted to communicate with other families who knew what they were going through and who could share what they had learned. They reported that their clinicians had difficulties providing prognoses, and so they turned to other families to compare children's developmental and health histories in hopes of finding similar cases that might shed light on the road ahead. One mother of a child with Trisomy $8 p$ was so anxious to determine whether her expectations for her son's developmental milestones were appropriate that she bought a computer as soon as her tax return arrived so that she could search the Internet for other Trisomy $8 \mathrm{p}$ families. She reported that she was very happy to find an online database of families having children with genetic conditions related to the eighth chromosome. She explained, "That's where I get more of my information, and then talking to families. So, I have a-you don't really get a timeline with this diagnosis-but, maybe kind of a ballpark."

In cases where parents did not agree with the medical geneticist's recommendations for treatment, the Internet became a way to locate "second opinions" from researchers, clinicians, and other families, as well as the research they would need to negotiate with their geneticists and other local healthcare providers about treatment options. For example, parents of children with Prader-Willi syndrome contacted other parents on the Internet, who urged them to push for the use of steroids and hormone treatments as early as possible. These parents reported that their doctors were reluctant to prescribe this treatment for young children unless the child was part of an experimental study, but influential success stories posted online prompted parents to continue arguing for earlier use of the hormone treatments. They attributed their success at obtaining this experimental treatment plan to the influential research compiled and disseminated by online parent advocacy networks.
For the majority of users, the Internet merely supplemented what they learned from their healthcare providers. But for those who felt they did not receive adequate information about their children's diagnosis and treatment options or that their concerns and ideas had not been taken seriously by doctors, the Internet became a primary source of information and support, as well as a forum through which avid users could advise other parents, raise funds for research and public awareness campaigns, and participate in other advocacyrelated efforts.

\section{Consequences of Internet use}

As parents went online to explore e-health resources, they entered numerous virtual, global communities formed around particular genetic disorders. These communities or online networks of parents and advocacy groups were influential in parents' decisions regarding treatment and diagnostic options. They validated families' decisions and provided them with resources that helped them become more proactive partners with service providers. As parents searched the Internet, explored personal home pages, and contacted other parents and experts, their scientific literacy grew and the value they placed on parental lay knowledge increased. Instead of viewing professionals as the sole provider of authoritative knowledge about their child's condition, parents came to see them as only one source of information and viewed themselves as critical information managers who were the arbitrators of that knowledge. They saw their role as seeking out and weighing all the information available to them, based not only on its perceived "scientific" merit but also on the extent to which it meshed with their own first-hand knowledge of their child.

A few parents took the information they had downloaded to their doctors or service providers for their perspective on it, but more often they communicated with other parents through chat rooms associated with specific disorders to help them assess the quality of information and advice and to learn what practices actually worked with their children. 
As parents discussed diagnostic and treatment options with their doctors, shared their experiences with other members of the online networks, and downloaded resources to share with family, friends, and service providers, they took part in the active co-construction of medical knowledge. Interpreting their diagnoses and related information, both for themselves and others, they helped determine what genetic knowledge means for families' daily lives.

Another consequence of the explosion of genetic knowledge and its availability on the Internet is that a genetic diagnosis has gained more value. A definitive genetic diagnosis provides a vital entryway to a world of information, and to specialists and groups organized around the disorder. With a diagnosis, a label, parents have a term to plug into search engines on the Internet, which guide them to a range of information about the basic genetics of the condition and possible treatments or interventions, and provide access to virtual research, advocacy, and support communities. Using a diagnosis to research a specific condition online, and then talking with teachers and other service providers so that they will better understand how to work with the child, was a common theme among families in this study. For example, a mother of a young girl with DiGeorge syndrome (a 22q deletion) bought a computer purely for the reason of researching this disorder. She found information on specific teaching strategies thought to be most effective for this condition, which she shared with her child's teachers. A mother of a child with Trisomy $8 p$ talked about keeping a binder of all the information she had found on this condition. She had used the Internet to research both genetic-based and symptom-based services and learned from her readings and communications via e-mail with other families that children with Trisomy $8 p$ do better when people use sign language rather than speech with them. She communicated regularly with 2 couples in England who had 2 older children with Trisomy 8 , and she followed carefully their experiences and the strategies they used. She also shared this in- formation with her child's doctors and early interventionists.

Parents' use of the Internet and the associated role of researcher and manager of information were not without drawbacks. Even those who said they benefited from their Internet research talked about the anxiety caused by online resources that contained contradictory advice, failed to answer their questions, painted a dire portrait of their child's future, or made them feel as though they had to keep looking to find the missing piece or link to information that would help their child. Avid users conveyed a heavy sense of responsibility, a moral obligation, to keep looking for information that might make a difference to their child's health and development. If only they could search harder and longer, they might find the crucial bit of information that could make a difference.

\section{IMPLICATIONS FOR EARLY INTERVENTION SPECIALISTS}

The conjunction of the Internet and genomics has important implications for early intervention. In speculations of how genetic discoveries will influence early intervention, scholars and practitioners have talked about how early interventionists will need to help families access and make sense of genetic information related to a disorder and possibly tailor interventions to the specific genetic disorder. Practitioners will no doubt have need for more information about basic genetics, genetic counseling, and supports and therapies related to specific disorders (Bailey, 2004; Bailey \& Powell, 2005; Hodapp et al., 2003), and indeed, our findings indicate that families search for this kind of information. Because of their Internet research and communications, parents believe they are more knowledgeable than most professionals about their child's particular genetic disorder. They have spent far more time doing the research and talking with other parents about optimal treatments and interventions, and they see their role as educating and negotiating with professionals 
about appropriate diagnoses or treatments. They become "citizen scientists" (Solovitch, 2001), who share authoritative knowledge with service providers. In fact, parents repeatedly referred to themselves as "experts" on their child's condition, and talked explicitly about the importance of being "on top of things" and "armed with research" in order to "make things happen" for their children. As one mother of a child with Prader-Willi syndrome said when asked what it would have been like for her without the Internet, "Oh my gosh. I cannot even imagine. I think I'd be taking what the doctors said more at face value ... I don't think he would have the advantage of me being able to sound assertive or aggressive, of going armed with information. I mean, it could make a major difference in his medical care."

The Internet, with its multiple points of entry to genetic information and communities, and the rapidly growing field of medical genetics, with its revolution in understandings of the molecular basis of disorders, are strong forces already impacting early intervention practices and relationships. As more genetic causes of disability are identified, families will increasingly be faced with learning about the genetic nature of their child's condition. How they access information and the kinds of information available have ramifications for all service providers and are likely to change how services are delivered as well as parent-professional relationships.

\section{REFERENCES}

Bailey, D. B. (2004). Newborn screening for fragile X syndrome. Mental Retardation and Developmental Disabilities Research Reviews, 10, 3-10.

Bailey, D. B., \& Powell, T. (2005). Assessing the information needs of families in early intervention. In M. J. Guralnick (Ed.), The developmental systems approach to early intervention (pp. 151-183). Baltimore: Paul H. Brookes.

Baker, L., Wagner, T. H., Singer, S., \& Bundorf, M. K. (2003). Use of the Internet and e-mail for health care information: Results from a national survey. JAMA, 289, 2400-2406.

Christian, S. M., Kieffer, S. A., \& Leonard, N. J. (2001). Medical genetics and patient use of the Internet. Clinical Genetics, 60, 232-236.

Collins, F. S., Green, E. D., Guttmacher, A. E., \& Guyer, M. S. (2003). A vision for the future of genomics research. Nature, 422, 1-13.

Condit, C. M. (1999). The meanings of the gene: Public debates about buman beredity. Madison: University of Wisconsin Press

Conrad, P., \& Gabe, J. (Eds.). (1999). Sociological perspectives on the new genetics. Oxford: Blackwell Publishers.

Cotten, S. R., \& Gupta, S. S. (2004). Characteristics of online and offline health information seekers and factors that discriminate between them. Social Science $\mathcal{E}$ Medicine, 59, 1795-1806.

CyberAtlas. (2001). Internet influencing all aspects of healthcare. Retrieved November 4, 2005, from http://www.webmetro.com/Newsdetail1. asp? $i d=642$

Emilien, G., Ponchon, M., Caldas, C., Isacson, O., \& Maloteaux, J. M. (2000). Impact of genomics on drug discovery and clinical medicine. Quarterly Journal of Medicine, 93, 391-423.

Falcon de Vargas, A. (2002). The Human Genome Project and its importance in clinical medicine. International Congress Series, 1237, 2-13.

Forness, S. R., \& Kavaale, K. A. (1994). The Balkanization of special education: Proliferation of categories for new behavioral disorders. Education and Treatment of Children, 17, 215-227.

Fox, S., \& Fallows, D. (2003). Internet bealth services. Washington, DC: Pew Internet \& American Life Project.

Gerber, B. S., \& Eiser, A. R. (2001). The patientphysician relationship in the Internet age: Future prospects and the research agenda. Journal of Medical Internet Research, 3(2), Article e15. Retrieved September 8, 2002, from http://www.jmir.org/2001/ 2/e15/index.htm

Giddens, A. (1991). Modernity and self-identity. Cambridge: Polity Press.

Hardey, M. (1999). Doctor in the house: The Internet as a source of lay health knowledge and the challenge to expertise. Sociology of Health \& Illness, 21, 820-835.

Hardey, M. (2000). The "home page" and the challenge to medicine. Health Information on the Internet, 16, 1-2. Retrieved November 8, 2002, from http://www.rsmpress.co.uk/hii/issue16.pdf

Health on the Net Foundation. (1999). 5th HON survey on the evolution of Internet use for health purposes. Retrieved November 8, 2002, from www.hon.ch/Survey/ResultsSummary_oct_nov99. html

Hodapp, R. M., DesJardin, J. L., \& Ricci, L. A. (2003). 
Genetic syndromes of mental retardation: Should they matter for the early interventionist? Infants \& Young Children, 16, 152-160.

Hodapp, R. M., \& Fiddler, D. J. (1999). Special education and genetics: Connections for the 21st century. Jour nal of Special Education, 33, 130-137.

Khoury, M. J., McCabe, L. L., \& McCabe, E. R. B. (2003). Population screening in the age of genomic medicine. New England Journal of Medicine, 348, 50-58.

Marion, R. W., \& Mendelson, A. (2000). First the bad news: What people with Marfan syndrome (MS) find when they search the Internet for information. American Journal of Human Genetics, 67, 203.

Miles, M. B., \& Huberman, A. M. (1994). Qualitative data analysis (2nd ed.). Thousand Oaks: Sage.

Nelkin, D., \& Lindee, M. S. (1995). The DNA mystique: The gene as a cultural icon. New York: W. H. Freedman.

Potts, H. W. W., \& Wyatt, J. C. (2002). Survey of doctors' experience of patients using the Internet. Jour- nal of Medical Internet Research, 4(1), Article e5. Retrieved September 8, 2002, from http://www.jmir. org/2002/1/e5

Skinner, D., Rodriguez, P., \& Baifey, D. (1999). Qualitative analysis of Latino parents' religious interpretations of their child's disability. Journal of Early Intervention, 22, 271-285.

Solovitch, S. (2001). The citizen scientists. Wired. Retrieved February 12, 2002, from http://www. wired.com/wired/archive/9.09/disease_pr.html

Taylor, M. R. G., Alman, A., \& Manchester, D. K. (2001). Use of the Internet by patients and their families to obtain genetics-related information. Mayo Clinic Proceedings, 76, 772-776.

Yoon, P., Chen, B., Faucett, A., Clyne, M., Gwinn, M., Lubin, I., et al. (2001). Public health impact of genetic tests at the end of the 20th century. Genetics in Medicine 3, 405-410.

Ziebland, S. (2004). The importance of being expert: The quest for cancer information on the Internet. Social Science \& Medicine, 59, 1783-1793.

Current articles- http://depts.washington.edu/isei/iyc/iyc_comments.html

Back to the list of previous articles:

http://depts.washington.edu/isei/iyc/iyc_previous.html 